\title{
Latex membranes with methylene blue dye for antimicrobial photodynamic therapy
}

\author{
Maria Andrelina O. Sousa ${ }^{1} \cdot$ Marco A. C. de Faria ${ }^{1} \cdot$ Rita P. Ribeiro $^{1} \cdot$ João V. P. Valverde ${ }^{1} \cdot$ Herica D. Rocha ${ }^{1}$. \\ Kevin F. dos Santos ${ }^{1} \cdot$ Marcos S. Sousa ${ }^{1} \cdot$ Paula C. S. Souto ${ }^{1} \cdot$ Josmary R. Silva ${ }^{1} \cdot$ Nara C. de Souza ${ }^{1}$
}

Received: 20 January 2021 / Accepted: 13 July 2021 / Published online: 22 July 2021

(c) The Author(s), under exclusive licence to European Photochemistry Association, European Society for Photobiology 2021

\begin{abstract}
The search for new materials that can be applied in the treatment of injured human tissues has led to the development of new dressings. Membranes have potential as dressing materials because they can be fitted to and interact with the tissue surface. In this study, we analyze the morphological properties and wettability of latex membranes, along with the incorporation of the photosensitizer methylene blue, in the context of the utility of the membranes in curative applications involving photodynamic therapy (PDT). It was observed that deposition of the photosensitizer into latex membranes increased both the surface roughness and wettability. Antifungal testing indicated that antimicrobial PDT assisted by the latex membranes incorporating methylene blue effectively inactivated Candida albicans.
\end{abstract}

Keywords Natural rubber $\cdot$ Burn $\cdot$ Microscopy $\cdot$ Wettability

\section{Introduction}

According to the World Health Organization, it is estimated that 300,000 deaths are caused by burns annually [1]. Burns are traumatic wounds that can cause partial or total destruction of the skin. In these situations, the skin is no longer able to maintain its function as a protective barrier for the body [2]. Treatments are designed to alleviate the systemic changes that result from the breakdown of this protective barrier [3]. Dressings that are used during recovery from burns must be able to maintain high humidity since the hydration of the wound facilitates the exchange of the dressing, thus aiding in the cicatrization process [4].

Among the candidate materials being investigated for the manufacture of dressings for burns, those based on natural rubber (NR) latex stand out for being highly flexible, translucent, and non-adhesive. These membranes have been investigated for regenerative medicine applications because of their angiogenic properties, high flexibility, and capacity for incorporating drugs into their structures [5-7], allowing the incorporation of photosensitizing dyes (PS) which are used

Nara C. de Souza

ncsouza.ufmt@gmail.com

1 Grupo de Materiais Nanoestruturados, Universidade Federal de Mato Grosso, Barra do Garças, Mato Grosso, Brazil in photodynamic therapy (PDT). Antimicrobial PDT is an alternative treatment for microbial infections [8] which uses a PS excited by light in the presence of molecular oxygen. After being excited, the PS can produce reactive oxygen species, resulting in the destruction of specific cells of a microorganism without damaging the surrounding tissues $[9,10]$.

According to Moore [11], septicemia due to Candida is a rare but important cause of death in burn patients. In burn injuries, colonization by Candida is a common cause of persistent infection. As the time and cost of the treatment of burn patients increases due to fungal infections, infected burns are a target of interest for antimicrobial PDT [8, 12].

The objective of this work was to analyze the morphological and wettability properties of latex membranes and to evaluate the effectiveness of antimicrobial PDT assisted by latex membranes incorporating methylene blue (MB) photosensitizer, thus investigating the potential of the membranes for use as curative membranes in PDT.

\section{Materials and methods}

The membranes were prepared as described by Silva [6]. Latex was extracted from a Hevea brasiliensis clone RRIM600 and stabilized with ammonium hydroxide. The NR membrane was obtained by drying the mixture in petri 
dishes at $50{ }^{\circ} \mathrm{C}$ for $96 \mathrm{~h}$. MB (Sigma Aldrich) was used as supplied. The NR membrane was immersed in the MB solution $\left(0.1 \mathrm{gL}^{-1}\right)$ for $4 \mathrm{~min}$ and then dried under ambient conditions (named NR+MB). Some membranes were stored in petri dishes wrapped with plastic film to avoid exposure to air, for 1 year. The packaged plates containing the membranes were protected from light during the storage period.

The membranes were monitored by a double-beam UV-vis spectrophotometer (Genesys 10, Thermo Scientific, Waltham, MA, USA). Surface morphology was analyzed by an atomic force microscope (AFM; EasyScan II, Nanosurf, Switzerland $)$ in the intermittent mode $(512 \times 512$ pixels $)$ with scanning windows of $5 \times 5 \mu \mathrm{m}$. The contact angle was measured with a homemade instrument under ambient conditions. The wetting contact angle analyzer is a compound of a monocular microscope and a positioner attachment to the microscope by clamps as described by Gomes [13]. Droplets of deionized water $(3 \mu \mathrm{L})$ were placed onto the membrane, and average values were taken from measurements at six different locations on each sample.

Inactivation tests were performed by spreading the dispersion containing Candida albicans (ATCC 14,053 at $10^{7} \mathrm{CFU} / \mathrm{mL}$ ) over the entire surface of the culture medium, and after $1 \mathrm{~h}$, membrane discs (diameter $0.6 \mathrm{~cm}$ ) were deposited on the medium. Discs were irradiated using a continuous diode laser (HLM1845) with a wavelength of $655 \mathrm{~nm}$. The output power of the laser beam was set to $100 \mathrm{~mW}$, using a spot size diameter of $\sim 0.6 \mathrm{~cm}$ and irradiation time of $85 \mathrm{~s}$, resulting in a dose of $30 \mathrm{~J} / \mathrm{cm}^{2}$. The laser beam output was positioned $15 \mathrm{~cm}$ from the top of a Sabouraud agar plate [14].The Petri dishes were incubated at $37^{\circ} \mathrm{C}$ for $24 \mathrm{~h}$. MB has already been acknowledged for the inactivation of $C$. albicans [15, 16]; hence, instead of classical tests, we used a variant of inhibition zone formation $[6,17]$ with the aim of verifying whether NR+MB avoided colonization of the fungus on the agar surface, which served as a proxy for the surface of burned skin.

For a quantitative analysis of the antifungal effect, we used the inactivation tests with adaptations. $80 \mu \mathrm{L}$ stock suspension of $C$. albicans $\left(10^{7} \mathrm{CFU} / \mathrm{mL}\right)$ and membrane discs (diameter $0.6 \mathrm{~cm}$ ) were placed in a microtube and after 5 min of contact, the material was irradiated and prepared for viability tests. This work used a viability assay based on the exclusion of the methylene blue dye [18-22], which acts as an indicator of cell viability. The counting in the Neubauer chamber was done with the help of the optical microscope, which considered the cells that were stained blue to be dead and the transparent ones to be viable [23].For morphological analysis, films with the dispersion of fungi were prepared by the casting technique [24], which involves the spreading of the material on a substrate. The entire procedure was performed in a laminar flow hood. The susceptibility of fungi was analyzed by fluorescence microscopy
(Nikon Eclipse $\mathrm{Ci} / \mathrm{L}$, Tokyo, Japan) using the acridine orange dye as marker, which fluoresces green when the cells are viable and red-orange when damaged [23, 25-29]. The microscope uses a mercury vapor lamp and for this experiment, the images were taken using a blue excitation filter (with dichroic barrier filter) which results in green, yellow or red emission. In this study at least three measurements for each sample were taken, and errors were determined as standard deviations. The groups were compared by ANOVA tests followed by a Tukey-Kramer multiple comparison test. Differences were considered significant at $p<0.05$.

\section{Results}

\subsection{Incorporation of MB}

The absorption at $670 \mathrm{~nm}$ for MB (Fig. 1) corresponded to monomers, and the shoulder at $610 \mathrm{~nm}$ was related to a vibrational transition. The MB dye was effectively deposited onto the latex membranes, as can be seen in the inset of Fig. 1. The NR membrane did not absorb in the region of the spectrum investigated.

\subsection{Wettability}

The contact angles, $\theta_{c}$, of the latex membrane decreased from $53^{\circ}$ to $19^{\circ}$ after MB deposition; i.e., there was an increase in wettability (Table 1) [13]. Two factors determine variations in the $\theta_{c}$ : chemical composition and surface morphology. The observed variations in $\theta_{c}$ may thus have been caused by changes to both the morphology and the composition [13] upon incorporation of MB into the NR.

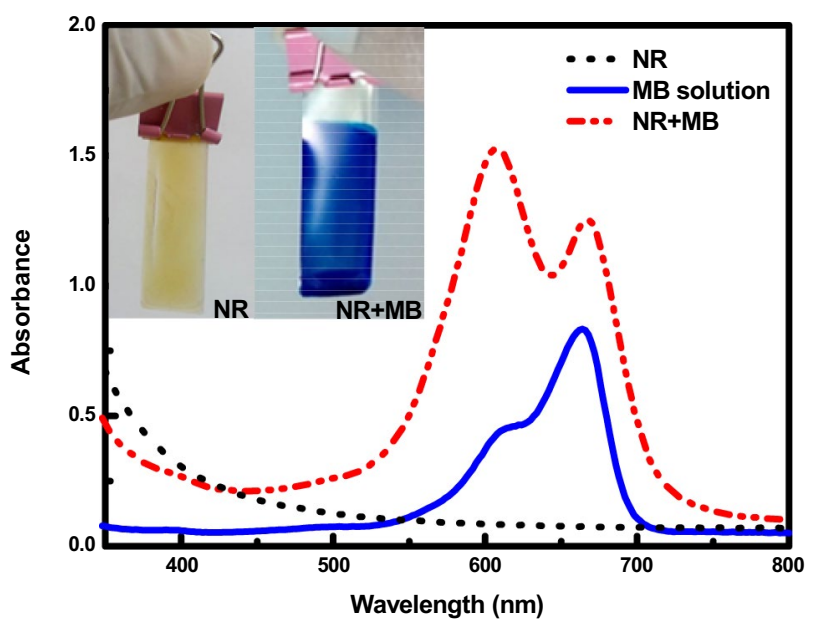

Fig. 1 UV-Vis spectra of MB solution and NR and NR+MB membranes. The inset shows the membranes based on NR and NR+MB 
Table 1 Values of contact angle $(\theta c)$, roughness $(W)$, fractal dimension $(D F)$, and roughness exponent $(\alpha)$

\begin{tabular}{lllll}
\hline Membrane & $\theta_{c}$ & $W(\mathrm{~nm})$ & $\mathrm{df}$ & $\alpha$ \\
\hline NR & $53.4^{\circ} \pm 5.6$ & 10.2 & 2.19 & 0.81 \\
NR+MB & $18.7^{\circ} \pm 3.0$ & 84.7 & 2.25 & 0.75 \\
\hline
\end{tabular}

\subsection{Morphological analysis}

Figure 2 shows images of the membranes along with their height profiles. NR+MB was composed of aggregates, as indicated by the results of UV-Vis spectroscopy (Fig. 1).

Studies suggest a strong correlation between the roughness and the wettability of a surface [30]. To establish a relationship between the morphology and the wettability of the membranes, the fractal dimension and roughness were analyzed together with the contact angle (Table 1). The roughness values were obtained using the microscope software NanoReport from Nanosurf (Switzerland). The roughness of the latex membrane increased upon deposition of MB.

\subsection{Antifungal susceptibility}

Tests for the inactivation of $C$. albicans were performed using the different membranes with and without laser irradiation. It can be seen that the NR membrane did not inhibit cell growth (Fig. 3a) even after irradiation (Fig. 3b). For the NR+MB membranes without irradiation (Fig. 3c), no fungal inactivation was observed. However, in the presence of laser irradiation (Fig. 3d), the NR+MB membranes exhibited zones of inhibition. The results were similar for freshly prepared membranes and those that had been stored for 12 months.

Figure 4 a shows the percentage of $C$. albicans that were viable as a function of different membranes with and without laser irradiation. In the control groups, we see that $C$. albicans is not susceptible to irradiation with a laser $(30 \mathrm{~J} /$ $\mathrm{cm}^{2}$ ) without the presence of MB. Although inactivation was not fully achieved, the cell viability of the fungus was significantly reduced in the presence of the $\mathrm{NR}+\mathrm{MB}+$ laser irradiation ( $\left.30 \mathrm{~J} / \mathrm{cm}^{2}\right)$. Fluorescence microscopy (Fig. 4b) associated with appropriate markers can be used to evaluate cell viability. The vital dye, acridine orange, enters cells, mixes with intact DNA, and fluoresces green; if damaged, the cells appear orange-red [24]. The images confirm the susceptibility results presented in Fig. 4a. With the filters used, autofluorescence of Candida albican and methylene blue is not verified. The corresponding images are presented in the supplementary material.

\section{Discussion}

When molecules from the dye solution are transferred to a membrane surface, aggregation can be observed through an analysis of displacements or the appearance of new peaks in the UV/Vis spectra [31]. For the NR + MB membrane, it was verified that the monomer-related peak was not displaced, but a new peak appeared at $600 \mathrm{~nm}$ which
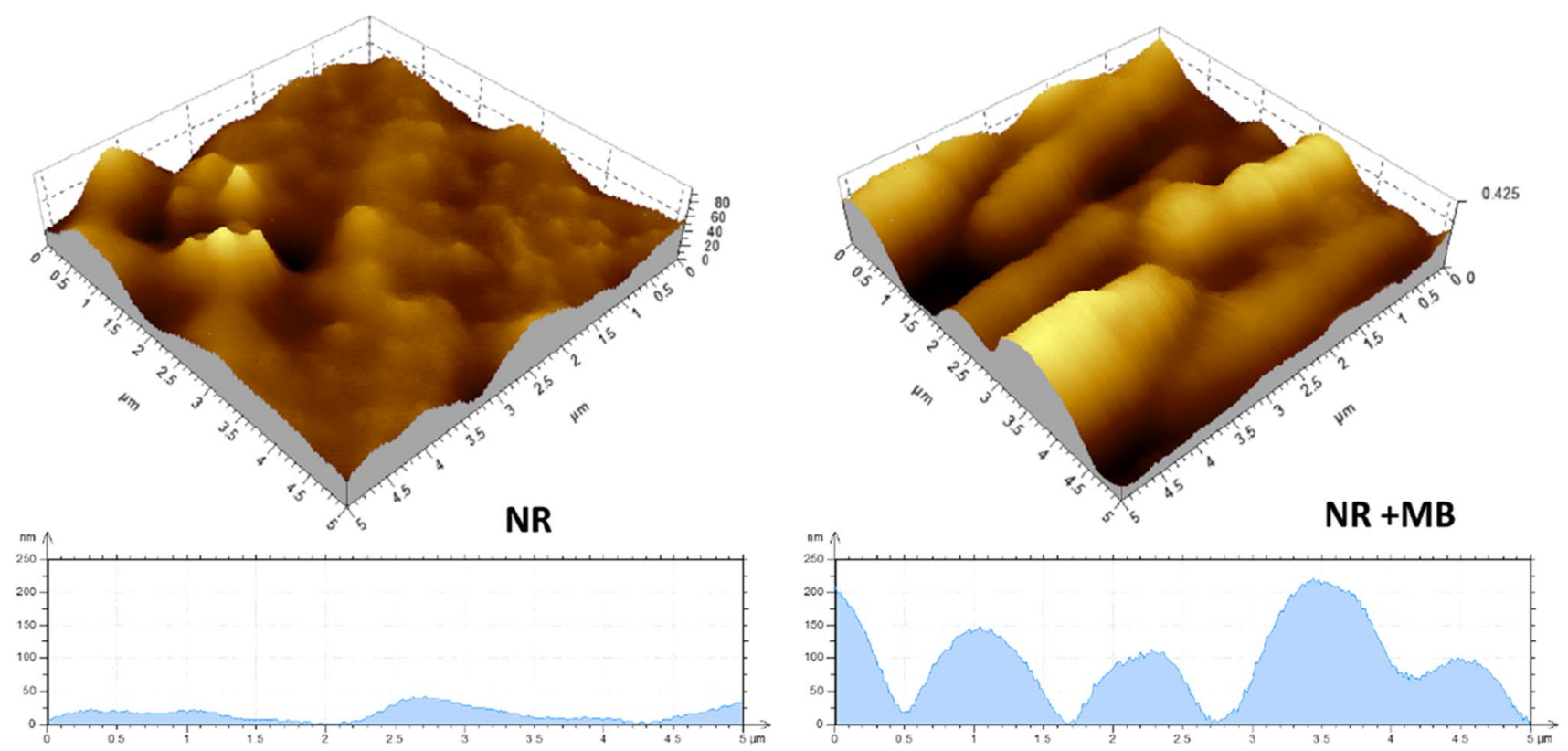

Fig. 2 AFM images of NR (left) and NR+MB (right) membranes. Height profiles were obtained from AFM images 
Fig. 3 a NR membrane and $\mathbf{b}$ NR membrane with $30 \mathrm{~J} / \mathrm{cm}^{2}$ irradiation, $\mathbf{c} \mathrm{NR}+\mathrm{MB}$ membrane and $\mathbf{d} \mathrm{NR}+\mathrm{MB}$ membrane with $30 \mathrm{~J} / \mathrm{cm}^{2}$ irradiation

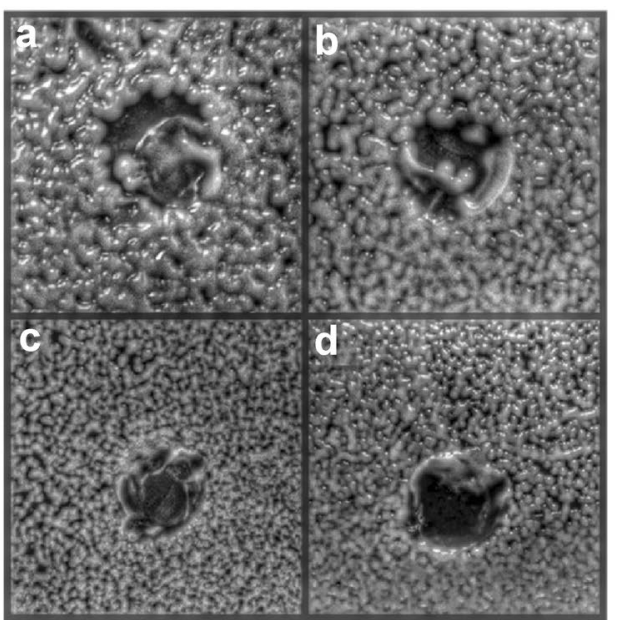

Newly prepared membrane

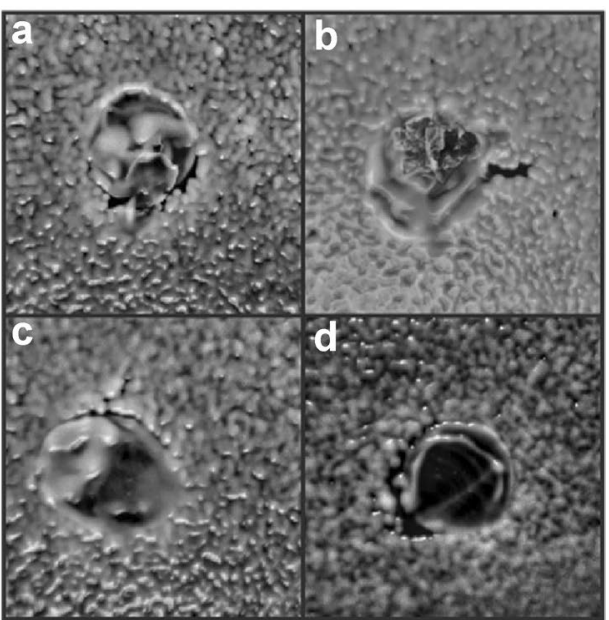

Membrane stored for one year a

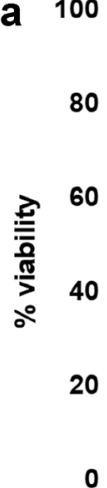

b
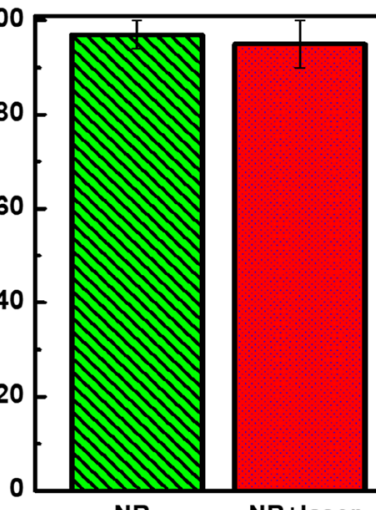

NR+laser
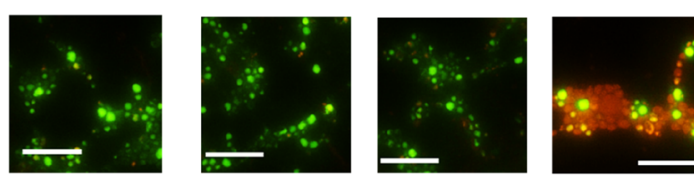

Fig. 4 a Cell viability of $C$. albicans dispersion. The control group is composed of an analysis of the dispersion of C. albicans +NR; irradiated $C$. albicans $+\mathrm{NR}\left(30 \mathrm{~J} / \mathrm{cm}^{2}\right) ; C$. albicans $+\mathrm{NR}+\mathrm{MB}$ (nonirradiated). The other results relate to the mixing of the $C$. albicans $+\mathrm{NR}+\mathrm{MB}$ irradiated $\left(30 \mathrm{~J} / \mathrm{cm}^{2}\right)$. Error bars represent the standard deviation. For each group 3 samples were analyzed and at least three measurements were taken. b Fluorescence microscopy of $C$. albicans dispersion. The dimension of the scale bar is $50 \mu \mathrm{m}$

was related to the formation of dimers during aggregation $[31,32]$. Aggregation can occur due to the high concentration of PS or the porosity of the membrane, which allows diffusion of the dye into the matrix volume [6, 33]. We recognize that the difference in energy excitation and geometry of monomers and aggregates must be considered when assessing the efficiency of the PS. However, studies have shown that MB dimers contribute to the type I and type II mechanisms of the photosensitization and photodestruction process of microorganisms [34, 35]. From our results, it is possible to assume that the presence of dimers should not be responsible for the decrease in PS efficiency. This question could be better answered, if the effect of different irradiation wavelengths were analyzed on the susceptibility of microorganisms. But these experiments were not conducted in this work.

Wettability is an important property when a surface is to be used for biomedical applications such as implants, sensors, or dressings, because the affinity of hydrophilic surfaces for the medium is generally stronger than that of hydrophobic surfaces [36, 37]. For example, higher wettability facilitates a stronger interaction between the surface of the dressing and the injured tissue. Increased wettability is an advantageous characteristic for dressings since these surfaces exhibit poor cellular adhesion to the new skin that is being formed during the cicatrization process; thus, using materials with higher wettability can minimize the pain and discomfort experienced during removal of the dressing [6, 38].

The relation between $\alpha$ and df of a surface can be obtained by: $\mathrm{df}=D-\alpha$, where $D$ is the dimension of the space observed [39]. The interface-forming process that determines the surface morphology can be investigated using fractal concepts and dynamic-scale laws [39, 40], which can be done by analyzing the log-log dependency of $W$ as a function of the scan window length (L). In the linear region, wherein the laws of scale are satisfied, the value of the angular coefficient gives $\alpha$ [39]. df values of $\sim 2$ are consistent with quasi-Euclidean surfaces associated with relatively flat interfaces [39]. It is reasonable to assume that a rearrangement of the molecules may occur in the $\mathrm{NR}+\mathrm{MB}$ membranes which minimizes the irregularities resulting from the random formation process of the surface, creating a less fractal structure. 
$W$ is determined by differences in height between the different points of the surface, whereas fractality is related to the way the roughness changes when the scale of observation changes [39]. Therefore, although the NR + MB membrane surface was rougher, the fractality was similar to that of the NR membranes. This can be clarified by analyzing the height profiles (Fig. 2). Since the height scale is the same, it is noted that at the same scanning windows $(\mathrm{L}=5 \mu \mathrm{m})$, the cross section of the NR + MB membrane was equally as fractal as the NR membrane, but the height variation between the peaks and valleys corresponding to the roughness was much larger for the NR $+\mathrm{MB}$ membrane.

Antifungal tests indicated that even with aging (Fig. 3), the NR $+\mathrm{MB}$ membrane did not lose its ability to inhibit cell growth. The result of irradiation $\left(30 \mathrm{~J} / \mathrm{cm}^{2}\right)$ of $C$. albicans dispersion mixed with $\mathrm{NR}+\mathrm{MB}$ showed that cell viability was reduced $(\sim 70 \%)$, confirming that membranes with the incorporation of the photosensitizer MB is effective for the photoinactivation of $C$. albicans (Fig. 4). The fluorescence images confirm the susceptibility results and indicate that the irradiation process had a fungicidal effect on C. albicans, although inactivation was not fully achieved with the parameters used. This result indicates that latex membranes incorporating $\mathrm{MB}$ can be used as antimicrobial dressings for the treatment of burns. These membranes are promising candidate materials for burn dressings since NR promotes angiogenesis and the combination of MB and laser irradiation exerts antimicrobial activity. As far as we know, this is the first time that an antimicrobial PDT is proposed using NR membranes incorporating a photosensitizer dye.

\section{Conclusion}

The incorporation of MB in latex membranes and the resultant changes were evaluated, opening opportunities to study the use of such materials as curative membranes with antimicrobial properties which are activated by light.

The deposition of MB in the latex membranes was responsible for increasing both surface roughness and wettability. It is suggested that for membranes with quasi-Euclidean surfaces, the adhesion between human skin and the dressing should be limited such that the membrane interacts with the injured tissue without excessive adhesion, thus minimizing the pain experienced during removal of the dressing. Antibacterial PDT tests indicated that the latex membrane incorporating MB was effective in inactivating C. albicans, which was used as a model microorganism. These results provide a starting point for research into curative membranes employing antimicrobial PDT.

Supplementary Information The online version contains supplementary material available at https://doi.org/10.1007/s43630-021-00077-z.
Acknowledgements This work was supported by CNPq (Brazil). Oliveira de Sousa, dos Santos, Sousa, de Faria, Ribeiro and Rocha would like to thank CAPES for the scholarship. Valverde would like to thank CNPq for the scholarship.

\section{Declarations}

Conflict of interest All authors certify that they have no affiliations with or involvement in any organization or entity with any financial interest or non-financial interest in the subject matter or materials discussed in this manuscript.

\section{References}

1. Burn prevention: success stories and lessons learned - World Health Organization - March 2011 (ISBN 978924150118 7). https://www.who.int/publications/i/item/9789241501187

2. Khoo, T. L., Halim, A. S., Saad, A. Z. M., \& Dorai, A. A. (2010). The application of glycerol-preserved skin allograft in the treatment of burn injuries: An analysis based on indications. Burns, 36, 897-904. https://doi.org/10.1016/j.burns.2009.03.007

3. Church, D., Elsayed, S., Reid, O., Winston, B., \& Lindsay, R. (2006). Burn wound infections. Clinical Microbiology Reviews, 19, 403-434. https://doi.org/10.1128/CMR.19.2.403-434.2006

4. Wasiak, J., Cleland, H., \& Campbell, F. (2008). Dressings for superficial and partial thickness burns. In J. Wasiak (Ed.), Cochrane database systematic reviews. Chichester: Wiley. https:// doi.org/10.1002/14651858.CD002106.pub3

5. Wattanakaroon, W., Akanitkul, P., Kaowkanya, W., \& Phoudee, W. (2017). Albumin-natural rubber latex composite as a dermal wound dressing. Materials Today: Proceedings, 4, 6633-6640. https://doi.org/10.1016/j.matpr.2017.06.178

6. Silva, A. J., Silva, J. R., de Souza, N. C., \& Souto, P. C. S. (2014). Membranes from latex with propolis for biomedical applications. Materials Letters, 116, 235-238. https://doi.org/10.1016/j.matlet. 2013.11.045

7. Krupp, T., dos Santos, B. D., Gama, L. A., Silva, J. R., ArraisSilva, W. W., de Souza, N. C., Américo, M. F., \& de Souza Souto, P. C. (2019). Natural rubber-propolis membrane improves wound healing in second-degree burning model. International Journal of Biological Macromolecules, 131, 980-988. https:// doi.org/10.1016/j.ijbiomac.2019.03.147

8. Jori, G., Fabris, C., Soncin, M., Ferro, S., Coppellotti, O., Dei, D., Fantetti, L., Chiti, G., \& Roncucci, G. (2006). Photodynamic therapy in the treatment of microbial infections: Basic principles and perspective applications. Lasers in Surgery and Medicine, 38, 468-481. https://doi.org/10.1002/lsm.20361

9. Mroz, P., Huang, Y., Szokalska, A., Zhiyentayev, T., Janjua, S., Nifli, A.-P., Sherwood, M. E., Ruzié, C., Borbas, K. E., Fan, D., Krayer, M., Balasubramanian, T., Yang, E., Kee, H., Kirmaier, C., Diers, J., Bocian, D., Holten, D., Lindsey, J. S., \& Hamblin, M. R. (2010). Stable synthetic bacteriochlorins overcome the resistance of melanoma to photodynamic therapy. The FASEB Journal, 24, 3160-3170. https://doi.org/10.1096/fj.09-152587

10. Choi, K.-H., Wang, K.-K., Oh, S.-L., Im, J.-E., Kim, B.-J., Park, J.-C., Choi, D., Kim, H.-K., \& Kim, Y.-R. (2010). Singlet oxygen generating nanolayer coatings on NiTi alloy for photodynamic application. Surface \& Coatings Technology, 205, S62-S67. https://doi.org/10.1016/j.surfcoat.2010.04.033

11. Moore, E. C., Padiglione, A. A., Wasiak, J., Paul, E., \& Cleland, H. (2010). Candida in burns: Risk factors and outcomes. Journal of Burn Care \& Research, 31, 257-263. https://doi.org/10.1097/ BCR.0b013e3181d0f536 
12. Acar, A., Uygur, F., Diktaş, H., Evinç, R., Lkür, E. Ü., Öncül, O., \& Görenek, L. (2011). Comparison of silver-coated dressing (ActicoatW), chlorhexidine acetate $0.5 \%$ (BactigrassW) and nystatin for topical antifungal effect in Candida albicans-contaminated, full-skin-thickness rat burn wounds. Burns, 37, 882-885. https:// doi.org/10.1016/j.burns.2011.01.024

13. Gomes, D. J. C., de Souza, N. C., \& Silva, J. R. (2013). Using a monocular optical microscope to assemble a wetting contact angle analyser. Measurement, 46, 3623-3627. https://doi.org/10.1016/j. measurement.2013.07.010

14. Costa Pedro, M. F., Kalck, A. S., dos Santos, K. F., Sousa, M. S., Romio, K. B., Souto, P. C. S., Silva, J. R., \& de Souza, N. C. (2018). Immobilization of triclosan and erythrosine in layer-bylayer films applied to inactivation of microorganisms. Photodiagnosis Photodynamic Therapy, 22, 158-165. https://doi.org/10. 1016/j.pdpdt.2018.04.012

15. Peloi, L. S., Soares, R. R. S., Biondo, C. E. G., Souza, V. R., \& Hioka, N. (2008). Elza Kimura, Photodynamic effect of lightemitting diode light on cell growth inhibition induced by methylene blue. Journal of Biosciences, 33, 231-237. https://doi.org/ 10.1007/s12038-008-0040-9

16. Tardivo, J. P., Giglio, A. D., de Oliveira, C. S., Gabrielli, D. S., Junqueira, H. C., Tada, D. B., Severino, D., de Fátima Turchiello, R., \& Baptista, M. S. (2005). Methylene blue in photodynamic therapy: From basic mechanisms to clinical applications. Photodiagnosis and Photodynamic Therapy, 2, 175-191. https://doi. org/10.1016/S1572-1000(05)00097-9

17. Pasquetti, M., Chiavassa, E., Tizzani, P., Danesi, P., \& APeano, (2015). Agar diffusion procedures for susceptibility testing of malassezia pachydermatis: evaluation of Mueller-Hinton agar plus $2 \%$ glucose and $0.5 \mu \mathrm{g} / \mathrm{ml}$ methylene blue as the test medium. Mycopathologia, 180, 153-158. https://doi.org/10.1007/ s11046-015-9913-2

18. Tolnai, S. (1975). A method for viable cell count. Tissue Culture Association Manual, 1, 37-38. https://doi.org/10.1007/BF009 14435

19. Chermsirivathana, S. (1952). A rapid method of staining for fungus and monilial infection. The Journal of Investigative Dermatology, 19, 7. https://doi.org/10.1038/jid.1952.60

20. Webb, R. J., Berger, L., Skerratt, L. F., \& Roberts, A. A. (2019). A rapid and inexpensive viability assay for zoospores and zoosporangia of Batrachochytrium dendrobatidis. Journal of Microbiological Methods, 165, 105688. https://doi.org/10.1016/j.mimet. 2019.105688

21. Ge, X., Gao, M., Situ, B., Feng, W., He, B., He, X., Li, S., Ou, Z., Zhong, Y., Lin, Y., Ye, X., Hu, X., Tang, B. Z., \& Zheng, L. (2020). One-step, rapid fluorescence sensing of fungal viability based on a bioprobe with aggregation-induced emission characteristics. Materials Chemistry Frontiers. https://doi.org/10.1039/ C9QM00732F

22. Kwolek-Mirek, M., \& Zadrag-Tecza, R. (2014). Comparison of methods used for assessing the viability and vitality of yeast cells. FEMS Yeast Research, 14, 1068. https://doi.org/10.1111/15671364.12202

23. dos Santos, K. F., Sousa, M. S., Valverde, J. V. P., Olivati, C. A., Souto, P. C. S., Silva, J. R., \& de Souza, N. C. (2019). Fractal analysis and mathematical models for the investigation of photothermal inactivation of Candida albicans using carbon nanotubes. Colloids and Surfaces B: Biointerfaces, 180, 393-400. https://doi. org/10.1016/j.colsurfb.2019.05.002

24. Romio, K. B., dos Santos, K. F., da Silva, R. J., Pedro, M. F. C., Kalck, A. S., da Silva Sousa, M., Possamai, L. M., Souto, P. C. S., Silva, J. R., \& de Souza, N. C. (2017). Incorporation of triclosan and acridine orange into liposomes for evaluating the susceptibility of Candida albicans. Journal Photochemistry Photobiology B: Biology, 173, 514-521. https://doi.org/10.1016/j.jphotobiol.2017. 06.034
25. Strugger, S. (1948). Fluorescence microscope examination of bacteria in soil. Canadian Journal Research, 26c(2), 188-193. https:// doi.org/10.1139/cjr48c-019

26. Guo, R., McGoverin, C., Swift, S., \& Vanholsbeeck, F. (2017). A rapid and low-cost estimation of bacteria counts in solutionusing fluorescence spectroscopy. Analytical and Bioanalytical Chemistry, 409, 3959-3967. https://doi.org/10.1007/s00216-017-0347-1

27. Martino, R. F., Davicino, R. C., Mattar, M. A., Casali, Y. A., Correa, S. G., \& Micalizzi, B. (2011). In vivo effect of three fractions of Larrea divaricata Cav (jarilla) on the innate immune system: Macrophage response against Candida albicans. Mycoses, 54, e718-e725. https://doi.org/10.1111/j.1439-0507.2010.02006.x

28. Paramanantham, P., Antony, A. P., Sruthil Lal, S. B., Sharan, A., Syed, A., Ahmed, M., Alarfaj, A. A., Busi, S., Maaza, M., \& Kaviyarasu, K. (2018). Antimicrobial photodynamic inactivation of fungal biofilm using amino functionalized mesoporus silicarose bengal nanoconjugate against Candida albicans. Scientific African. https://doi.org/10.1016/j.sciaf.2018.e00007

29. Chick, E. W. (1961). Acridine orange fluorescent stain for fungi. Archives of Dermatology, 83, 305-309. https://doi.org/10.1001/ archderm.1961.01580080135015

30. Denny, M. W. (2008). The Intrigue of the Interface. Science, 320, 931-934. https://doi.org/10.1126/science.1156023

31. Petty, M. C. (1996). Langmuir-Blodgett films : An introduction (p. 234). Cambridge: Cambridge University Press.

32. de Souza, N. C., Flores, J. C. J., \& Silva, J. R. (2009). Layerby-layer films from tartrazine dye with bovine serum albumin. Chemical Physics Letters, 484(1-3), 33-36. https://doi.org/10. 1016/j.cplett.2009.10.065

33. de Souza, N. C., Cavalheri, A. S., Brito, J. B., Job, A. E., Oliveira, O. N., Jr., Giacometti, J. A., \& Silva, J. R. (2012). Photoinduced orientation in natural rubber. Chemical Physics Letters, 531, 110-113. https://doi.org/10.1016/j.cplett.2012.01.070

34. Usacheva, M. N., Teichert, M. C., \& Biel, M. A. (2003). The role of the methylene blue and toluidine blue monomers and dimers in the photoinactivation of bacteria. Journal of Photochemistry and Photobiology B: Biology, 71(1-3), 87-98. https://doi.org/10. 1016/j.jphotobiol.2003.06.002

35. Bartlett, J. A., \& Indig, G. L. (1999). Effect of self-association and protein binding on the photochemical reactivity of triarylmethanes. Implications of noncovalent interactions on the competition between photosensitization mechanisms type I and type II. Photochemistry and Photobiology, 70(4), 490-498. https://doi. org/10.1111/j.1751-1097.1999.tb08243.x

36. Shin, S., Seo, J., Han, H., Kang, S., Kim, H., \& Lee, T. (2016). Bio-Inspired extreme wetting surfaces for biomedical applications. Materials, 9, 116-141. https://doi.org/10.3390/ma9020116

37. Falde, E. J., Yohe, S. T., Colson, Y. L., \& Grinstaff, M. W. (2016). Superhydrophobic materials for biomedical applications. Biomaterials, 104, 87-103. https://doi.org/10.1016/j.biomaterials.2016. 06.050

38. Kamoun, E. A., Kenawy, E. R. S., \& Chen, X. (2017). A review on polymeric hydrogel membranes for wound dressing applications: PVA-based hydrogel dressings. Journal of Advanced Research, 8(3), 217-233. https://doi.org/10.1016/j.jare.2017.01.005

39. Barabási, A.-L., \& Stanley, H. E. (1995). Fractal concepts in surface growth. New York: Press Syndicate of the University of Cambridge.

40. Gorza, F. D. S., da Silva, R. J., Trescher, T. F., Pedro, G. C., de Sousa, M. A. O., Souto, P. C. S., Silva, J. R., \& de Souza, N. C. (2016). Immobilization of chlorophyll by using layer-by-layer technique for controlled release systems and photodynamic inactivation. Photodiagnosis and Photodynamic Therapy, 15, 147-155. https://doi.org/10.1016/j.pdpdt.2016.06.010 